Disclosures: M. Perkowski, None.

Objective: To present an unusual case of neurogenic cramp disorder.

Patients or Programs: A 61-year-old woman with diagnosis of fibromyalgia presented to clinic with chronic history of back pain and muscle cramps.

Program Description: Patient describes both muscle spasms and muscle stiffness that developed over many years. Muscle spasms are diffuse and all over the body. This cramp causes her severe pain (7 of 10) and associated with diaphoresis. The spasms occur both during the day and night intermittently. She also complains of frequent falls due to her legs giving out, cannot walk 50 feet without profusely sweating, tires easily, paresthesia of bilateral feet, and has sleep difficulties. She started herself on high dose magnesium, $3200 \mathrm{mg}$ daily. She had been advised to stop taking high dose magnesium for concern of cardiac complications so she decreased her dose to $2400 \mathrm{mg}$ daily. She was diagnosed as having fibromyalgia and was treated with Lyrica. She developed adverse effects, the Lyrica was stopped, and she was started on Cymbalta, which improved her symptoms. She later stopped Cymbalta because she was gaining weight. After stopping Cymbalta she lost approximately 25 pounds and her pain remained under control with acetaminophen as needed. She had trialed physical therapy with no improvement. A neurology consult was obtained and the findings were muscles strength $4 / 5$, intact normal gait but difficulty toe and heel-walking, intact sensation, absent lower extremity reflexes, and bilateral and symmetric tenderness to palpation of interscapular region, shoulders, elbows, wrists, hips, knees, and ankles. Overall examination findings were not consistent with her diagnosis of fibromyalgia. EMG studies revealed peripheral neuropathy. Laboratory tests were obtained and she was diagnosed with a neurogenic cramp disorder exacerbated by neuropathy. It was recommended that she continue treatment with magnesium.

Setting: Outpatient PM\&R practice.

Results: A case of neurogenic cramp disorder mistakenly diagnosed as fibromyalgia.

Discussion: Fibromyalgia is a diagnosis by exclusion and muscle pain, spasms, fatigue, and insomnia are presenting symptoms. However, this symptom complex needs thorough investigation to rule out other organic diseases.

Conclusions: Proper diagnosis is the key for pain management.

\section{Poster 276}

Atypical Radiculopathy Secondary to Foraminal Stenosis Presenting as Restless Legs Syndrome: A Case Report.

Patrick W. Russell, MD (Medical College of Wisconsin, Milwaukee, WI); David deDianous, MD.

Disclosures: P. W. Russell, None.
Patients or Programs: A 54-year-old man with history of restless legs syndrome.

Program Description: The patient presented with bilateral anterior thigh and pretibial pain with paresthesias. Symptoms were worse at night and with prolonged sitting. Symptoms were improved with movement or shaking of the limbs. There was no increase in symptoms with standing or walking. Ropinirole had been less effective over the past several months. There was no pain with active lumbar range of motion and was no tenderness to palpation. Strength, sensation to light touch, and reflexes were intact. Straight leg raise was negative. After brief trials of gabapentin and pregabalin, lumbar spine MRI was obtained and revealed multilevel degenerative disk disease with mild central stenosis at L3-4 and foraminal stenosis at L3-4 and L4-5. The patient received bilateral L4 transforaminal epidural injections. At 2-week follow-up, the patient reported a significant reduction in pain and in restless legs. 8 months later, the patient returned with nocturnal paresthesias and resultant insomnia, excessive daytime fatigue, and poor work productivity. He denied low back or leg pain. The patient received repeated bilateral L4 selective nerve root blocks.

Setting: Outpatient comprehensive spine care clinic.

Results: At 2-week follow-up, the patient reported 80\%90\% improvement in nocturnal symptoms with resultant increase in sleep duration and work performance.

Discussion: Patients with atypical radiculopathy secondary to spinal stenosis can present similarly to patients with restless legs syndrome. This case highlights the necessity of excluding pathology that can mimic restless legs syndrome before medical treatment.

Conclusions: When treating patients for restless legs syndrome it is wise to consider alternative etiologies, such as spinal stenosis and radiculopathy before finalizing treatment plans.

\section{Poster 277}

\section{Cervical Transforaminal Epidural Steroid Injection in the Management of a Stinger Injury: A Case Report.}

Jared Greenberg, MD (University of Michigan, Ann Arbor, MI); Daniel Leung, DO; Michael J. Louwers, MD.

Disclosures: J. Greenberg, None.

Patients or Programs: A 21-year-old male collegiate football player with a history of multiple stingers.

Program Description: The patient presented to clinic with 5 days of persistent left-sided neck and shoulder pain associated with upper extremity paresthesias and weakness. His symptoms began during a game, after being struck on the right side of his helmet, inducing left lateral flexion, and extension of his neck. Examination and electrodiagnostic evaluation were consistent with an acute C5 radiculopathy. Cervical spine MRI was unremarkable, further supporting a 
diagnosis of stinger injury. Because his symptoms had not improved with time and conservative therapies, and prompt return to play was desired, a cervical transforaminal epidural steroid injection was offered.

Setting: University outpatient clinic.

Results: Within 1 week after the injection, the patient had full resolution of his pain symptoms and neurologic dysfunction, and he was cleared for return to play.

Discussion: A "stinger" or "burner" is a transient, reversible peripheral nerve injury of the upper extremity caused by injury to the cervical spine and shoulder, usually during contact sports. It generally affects the upper trunk or C5/C6 nerve roots. To our knowledge, this is the first reported case of a stinger injury to be treated with a cervical transforaminal epidural steroid injection. It is believed steroids decrease inflammation, improve regional blood flow, and become neuroprotective by inhibiting phospholipase A2 and lipid peroxidation. A transforaminal approach should be considered as it typically produces proximal and distal nerve root spread to deliver injectate along the suspected pathology.

Conclusions: Although cervical injections are not without risk, they may be of great benefit to patients with "chronic stinger syndrome" that have failed conservative treatment. Further investigation is needed to substantiate the benefits of this treatment approach.

\section{Poster 278}

\section{Complex Regional Pain Syndrome Type II in the Setting of Lumbosacral Plexopathy:} A Case Report.

\section{Andrew Gallo, DO (Walter Reed Army Medical} Center, Washington, DC); Vincent T. Codispoti, MD.

Disclosures: A. Gallo, None.

Patients or Programs: A 25-year-old male polytrauma patient.

Program Description: A 25-year-old male soldier was injured in a blast injury sustaining multiple spinal compression fractures and left pelvis and sacral fractures. In theatre, he underwent external pelvic fixation. The surgeons noted traction on the lumbosacral plexus due to the fractures. He was transferred to a U.S. Army medical center for further surgery and inpatient rehabilitation. Approximately 3 weeks into his course, he complained of swelling and burning pain in the left ankle and foot. His evaluation was significant for weakness and diminished sensation in the left lower extremity corresponding to multiple myotomes and peripheral nerves. There was hyperesthesia, allodynia, and edema of the left ankle and foot without point tenderness. Skin temperature and texture was normal. Plain films of the left leg were negative. EMG/NCS revealed evidence of a left lumbosacral plexopathy, involving all portions of the plexus as well as probable injury at the nerve roots or cauda equina. We could not obtain MRI due to the orthopedic hardware but CT of the left ankle and foot showed no bony or significant soft tissue abnormality. A bone scan showed findings consistent with complex regional pain syndrome.

Setting: Inpatient rehabilitation unit of a military hospital. Results: The patient was treated with aggressive physical therapy, modalities, and medications to include pregabalin, nortriptyline, prednisone, and morphine with $75 \%$ relief. His pain continued to improve and he was eventually able to walk independently when his pelvic external fixation device was removed.

Discussion: To our knowledge, this is only the second case of complex regional pain syndrome (CRPS) type II described in the setting of lumbosacral plexopathy.

Conclusions: Although CRPS type II has classically been described in the setting of mononeuropathy, it is important to keep in the differential diagnosis of neuropathic pain in the setting of known nerve injury to achieve the best functional outcome.

\section{Poster 279}

Gluteal Lipoma Producing Radicular Pain. Debora Mottahedeh, DO (The Mount Sinai Hospital, New York, NY); Houman Danesh; Parag Sheth, MD.

Disclosures: D. Mottahedeh, None.

Patients or Programs: A 50-year-old African American woman with radicular pain.

Program Description: Process of evaluation and elucidation of symptomatic radicular pain.

Setting: University hospital.

Results: Pain reduction of $80 \%$ after surgery, using the visual analog scale.

Discussion: Lipomas are the most common soft-tissue tumor, occurring in $1 \%$ of the population and are most often asymptomatic. They are slow-growing, benign fatty masses enclosed by a thin, fibrous capsule. Nerve compression by a lipoma is uncommon but does occur. Lipomas are capable of creating neural compression by either enveloping the nerve or being in close approximation in a confined space. This compression can occur at a point where the nerve runs superficially or the lesion can be derived by deeper-seated fatty tissue, which is less frequent. The most frequent clinical presentation of these tumors is pain. For superficial lipomas that can be palpated, positive Tinel sign over the lesion is the most useful sign to help identify the tumor as the source of nerve compression. The situation is different for deeper tumors. These lesions often reach a significant size before clinical presentation due to the potential space of the retroperitoneum. Often, such masses mimic other musculoskeletal syndromes. Resection of the lipoma usually results in resolution of symptoms. Electromyographic studies obtained preoperatively can provide guidance for the intended surgical approach. In patients with compromised neural function, the tumor may be resected along with associated neural elements. 